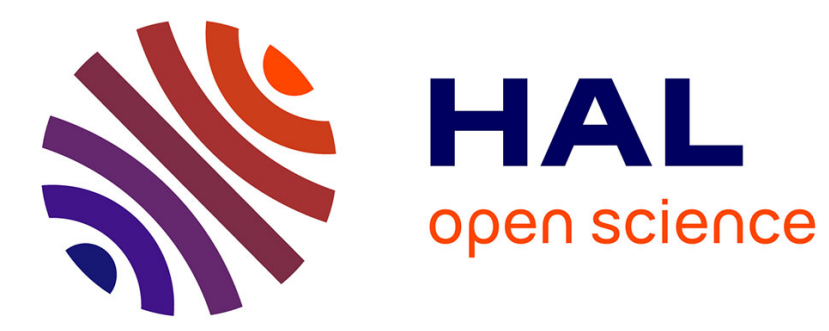

\title{
Beyond Full Jurisdiction: Pathology and Inter-Professional Relations in Precision Medicine
}

Henri Bergeron, Patrick Castel, Audrey Vézian

\section{To cite this version:}

Henri Bergeron, Patrick Castel, Audrey Vézian. Beyond Full Jurisdiction: Pathology and InterProfessional Relations in Precision Medicine. New Genetics and Society, 2021, Organizing Precision Oncology, 40 (1), pp.42-57. 10.1080/14636778.2020.1861543 . halshs-03091473

\section{HAL Id: halshs-03091473 \\ https://shs.hal.science/halshs-03091473}

Submitted on 3 Jan 2022

HAL is a multi-disciplinary open access archive for the deposit and dissemination of scientific research documents, whether they are published or not. The documents may come from teaching and research institutions in France or abroad, or from public or private research centers.
L'archive ouverte pluridisciplinaire HAL, est destinée au dépôt et à la diffusion de documents scientifiques de niveau recherche, publiés ou non, émanant des établissements d'enseignement et de recherche français ou étrangers, des laboratoires publics ou privés. 


\section{Beyond Full Jurisdiction: Pathology and Inter-professional Relations in Precision Medicine}

\section{Bergeron Henri}

Sciences Po, CSO

\section{Castel Patrick}

Sciences Po, CSO

\section{Vézian Audrey}

Triangle, CNRS, ENS Lyon)

\section{Correspondance:}

Sciences Po, CSO, 27 rue Saint-Guillaume, 75007 Paris

Email: Patrick.castel@sciencespo.fr

Henri Bergeron is a Senior CNRS Research Fellow at the Center for Sociology of Organizations, Sciences Po, co-director of the Health Department of Interdisciplinary Centre for the Evaluation of Public Policies (Centre of Excellence - LABEX), and Scientific coordinator of the Chair in Health Studies-Sciences Po. He is also the director of the Master on "Organizational behavior and Human Resources" at Sciences Po and the Scientific Director of the "Management and Public Affairs" track at Sciences Po's School of Public Affairs. He teaches and conducts research on organizational behavior, institutional changes and drivers (including digital transformation), on institutional entrepreneurship, and leadership, and on power in and between organizations.

Two main themes run through Patrick Castel's research: the organization of medical activities and the processes of institutional change involved in this, and the processes of designing and implementing public healthcare policies (especially, obesity). He has worked on the organization of patient management for cancer patients, the processes by which recommendations for clinical practice are 
produced and implemented, and the organization of clinical and translational research. He has published articles in academic journals, such as Organization science, Social studies of science, The Lancet, Social Science \& Medicine.

Audrey Vézian's research focuses on the implementation of biomedical policies. Taking the creation of new regional, public entities as an example, her $\mathrm{PhD}$ examined the role of research administration practices in the spread of biomedicine in France. Currently, her work has focused on the potential of big data in health contexts, and their consequences on the organization of medical activities. 


\title{
Beyond Full Jurisdiction: Pathology and Inter-professional Relations in Precision Medicine
}

\begin{abstract}
The rise of precision medicine represents a challenge for pathology, which must now more closely link research and diagnostic and collaborate on new bases with other specialties. Our paper is based on the study of four French cancer centers invested in developing precision medicine. Molecular biology is particularly threatening for pathologists in settings where medical oncologists and biologists believe that the discipline is capable of presiding over if not altogether replacing morphology as the principal basis for diagnosis. However, we will show that the organizational arrangements for circulating tumor specimens and producing diagnoses, as well as the place occupied by pathologists in these arrangements, have varied from place to place and over time. These configurations are the result of preexisting arrangements and local negotiations among professionals, where the aim is rather to include and coordinate specialties within the production line rather than exclude them from the jurisdictional remit.
\end{abstract}

Key words: cancer, profession, pathology, cooperation, translational research

\section{Introduction}

As is the case with innovation anywhere, the effective deployment of genomics-derived methods and techniques requires changes in practices, in professional relations and, more generally, in the local organization of healthcare services. Recent research has investigated the changes in the doctor-patient relationship and the difficulties inherent to these changes when implementing precision medicine in routine care (Kerr et al. 2019). The profound changes in the division of labor among professionals in the production of routine genomic diagnostics are also beginning to be explored (Beaudevain, Peerbaye, and Bourgain 2018; Wright et al. 2019).

Such transformations have also become necessary in the context of translational research, and in some respects they are perhaps even more complex here. Indeed, not only 
does the production of knowledge in this context require the cooperation of many research actors and the mobilization of many instruments, devices, and facilities; it also requires cooperation between healthcare and research actors, contributing to a "fading boundary between research and care" (Cambrosio et al. 2018). Bourret and Cambrosio (2019) recently investigated the emergence of a new form of collective expertise in the case of molecular tumor boards, which is a new organizational entity.

While pathology has been the specialty at the heart of cancer diagnosis for more than a century, the way in which pathologists contribute to the production of new knowledge in relation to precision medicine and the transformations in their practices and work organization that this involves have been less studied. The current article intends to bridge this knowledge gap. It draws on the study of four French cancer centers invested in the development of precision medicine, in particular through the deployment of genomic-driven trials. We observed how the circulation of tumor specimens and the production of diagnoses were locally organized and how pathologists intervened in these processes in relation to other actors.

For a long time, access to specimens and the production of a diagnosis have lain within the jurisdiction of pathology, and a kind of monopoly on this process has been enjoyed by its practitioners. Furthermore, pathologists are still legally responsible for the management and storage of tumor specimens. The growth of precision medicine has represented a challenge for this medical specialty, as it must now more closely link research and diagnostic practices and collaborate on new bases with other specialties and disciplines, including, in particular, medical oncology, molecular biology, and bioinformatics. A further challenge lies in the need to connect knowledge and taxonomies that pathology as a discipline has thus far mastered to other knowledge and taxonomies related to precision medicine (Green et al. 2019). 
In this context, it is tempting to apply a "doing lens" (Anteby et al. 2016), a framework shared by researchers within both the sociology of science and the sociology of professions, according to which professional, disciplinary, and expert groups struggle amongst themselves to reserve domains, defending "boundaries" (Amsterdamska 2005; Lamont and Molnar 2002) and even controlling "monopolies" (Abbott 1988; Gieryn 1983). According to this "lens", hierarchies between professional groups are clearly defined (see, e.g., Crabu [2018] for a recent review on translational research). In some settings, the jurisdiction of molecular biology has posed a particular threat for pathologists, where medical oncologists or biologists believe that their knowledge is in a position to preside over if not altogether replace morphology as the main basis for diagnosis. We shall see, however, that such an analytical grid is too reductive, insofar as it fails to account for the variety of situations observed and is incapable of providing a satisfactory interpretation of why and how actors collaborate across so-called jurisdictions, even in a conflictual manner.

Rather than hypothesize on the nature of a special ethos proper to pathologists (“doctors" vs. "researchers") (very early, Bucher (1962) distinguished two segments among pathologists between scientific-oriented pathologists vs practitioners), we will show that their degree of involvement in genomics and the conflicts they come into with other professionals around precision medicine can be explained by a) the kinds of exchanges that take place between clinicians, biologists, and pathologists over the division of labor; b) the challenge of integrating the new tasks related to research without disturbing the routinized and rationalized organization of work related to care; c) the need to maintain good relationships with other (private) pathologists. Pathologists' engagement in precision medicine, or lack thereof, has less to do with an ethos than with their need to maintain stable networks of cooperation with other actors, without whom they cannot fulfill their work. 
An analysis of tensions related to precision medicine inside pathology laboratories makes possible a genuine understanding of the interconnectedness of care and research, already identified in other professional health areas (Nelson et al. 2014). It makes it possible to examine the way in which genomics shapes the organization of routine care activities but also, reciprocally, how the imperatives proper to care, which are based on complex collaborations between professionals, challenge the innovative epistemic process involved in genomics research.

\section{Settings and methods}

Conceived as a key measure of the Cancer Plans 2009-2013 and 2014-2019, the French

National Cancer Institute accredited eight sites to implement "translational research" against cancer i.e. to "optimize and accelerate the production of new knowledge and promote its dissemination and application in the treatment of cancers". On these sites, cancer genomics is a privileged topic of translational research, though investment in genomics has varied in kind and depth from site to site, and has tended over the years to be weighed up against immunology/ immunotherapy. The sites also have in common the allocation of substantial financial and human resources to the creation or strengthening of common platforms, made available to the entire local professional community, and linked to precision medicine, such as Next Generation Sequencing, Array comparative genomic hybridization, and bioinformatics.

This study is based on 90 qualitative interviews conducted between 2013 and 2017 with different types of actors (pathologists, clinicians, biologists, bioinformaticians, etc.) in four of these sites. These sites were selected to represent contrasting contexts. Two of them (A \& B) were located within a single cancer centre, each one being considered as a top leading organization in translational research before the accreditation. A third one $(\mathrm{C})$ brought together research teams and practitioners from a cancer centre and a university hospital; both 
health organizations had a visible activity in clinical research and some basic research areas, but their reputation was not as strong as the A \& B. The fourth one (D), which also brought together researchers and practitioners from a cancer centre and a university hospital, did not have a strong reputation in translational research before accreditation and might be considered as a challenger in the field. Despite these differences between the sites, we will see that our findings on the difficult integration of pathologists in precision medicine projects and the conditions for cooperation are common features.

\section{A specialty under pressure in the context of precision medicine}

On all these sites, the integration of pathologists in the deployment of translational research projects related to molecular biology was a recurring subject of discussion and even, in some cases, conflict. This was particularly the case for novel kinds of clinical trials, which were designed to validate genomic biomarkers and evaluate targeted therapies. On the sites A and $\mathrm{C}$, for example, clinician-researchers and biologists involved in these trials attempted to base therapeutic decisions solely on the molecular characterization of tumors and by so doing, to bypass pathologists' expertise. In an additional sign of neglect, compared to the resources allocated to biology departments, some of the clinician-researchers in charge of these translational research projects allocated the corresponding pathology departments very few resources to help them to participate in the development of precision medicine.

[Pathologists] stick to their notion of diagnosis. [...] In the reports they come out with, it's really a complete diagnosis. But, actually, we don't need it! [...] The only criterion that's important to us is the tumor percentage. Period.

Interview with a biologist in charge of an NGS platform

Beyond these specific cases, most of the pathologists we interviewed testified to recurrent difficulties in relation to translational research projects in precision medicine and, more generally, to a lack of symbolic recognition on the part of the other actors involved in these 
types of project. This feeling was widely shared, as one of us was able to observe by attending six meetings of the French Association for Quality Assurance in Anatomo-Cytopathology and all the meetings of the union of French pathologists between 2017 and 2019.

I think the profession of pathologist is not necessarily well understood by our colleagues, the clinicians. Surgeons understand our work better, because we work with them a lot. But oncologists are quite new to this type of work, this type of collaboration, because they now need pathologists' samples to be able to do their genomic analysis.

Interview with a pathologist

Reciprocally, the medical oncologists who attempted to design and implement translational research projects and particularly genomic-driven trials expressed a common set of criticisms towards pathologists. They reproached them for a lack of involvement in research activities and for prioritizing their routine diagnostic activity, which was reflected in delays in processing and in making tumor specimens available for biologists and oncologists as well as in reporting histopathological analyses, in their lack of involvement in molecular diagnostics, and in the lack of standardization of reporting.

It's a difficult discipline. Well, difficult for itself, I would say. Which has had a hard time accepting change. [...] But it is a discipline that is so central to the process that this difficulty has become visible. We haven't solved our problem with the local pathologists at all. Not at all. It's one of our key problems.

Interview with a medical oncologist.

In order to alleviate these difficulties, the pathology community undertook various collective actions. They aimed in particular to defend the idea that pathologists had a role to play as integrators of clinical and molecular data in the implementation of targeted therapies. ${ }^{1}$ They

\footnotetext{
${ }^{1}$ See for instance the "Declaration on Molecular Pathology" by the Section of Pathology of the European Union of Medical Specialists, at the end of their annual meeting, in Madrid, June $8^{\text {th }} 2013$ (https://www.uems.eu/_data/assets/pdf_file/0005/7556/Item-5.3.6-Section-Pathology-MadridDeclaration-en.pdf). This declaration, which included the statement that "[a]s Pathology is the specialty that is best placed to maximise the use of tissue and cellular samples, molecular Pathology
} 
also participated in national programs to improve the quality of molecular genetic exams on tumor specimens. We also observed some capacity for pathologists to intervene in the local organization of precision medicine projects. For example, on one of the sites we studied, faced with what they considered an inconsiderate work overload due to the additional analyses of tumor specimens required for a precision medicine trial, pathologists mobilized and obtained a three month suspension of patient accrual to this trial from the head management of the cancer center. The principal investigator of the trial was furious but had to accept this decision and find alternative organizational solutions for the continuation of the trial after the pause, as we will see later.

\section{A pairing between pathologist and molecular biologist is nonetheless unavoidable}

Confronted with these conflicting situations, research actors tended to provide a simple explanation for the reluctance of pathologists to fully participate in the development of precision medicine. They tended to explain this, in the way that some sociologists might do, as the result of differences in ethos between researchers and practitioners ${ }^{2}$ (see, e.g., Löwy 1996; Kerr et al. 2019) - pathologists being above all doctors and not researchers (cf. infra) and/or as reactions in defence of a jurisdictional space threatened by genomic medicine and its promoters (Wilson-Kovacs and Hauskeller 2012; Hendriks, Simons, and Reinhart 2019; Kerr et al. 2019). We maintain, however, that these explanations are unsatisfactory.

\section{A hitherto unavoidable position}

There is no doubt that pathologists feared their prevailing authority in diagnosis, dearly won at the beginning of the 20th century (Close-Koenig 2007), being called into question. Indeed,

must be performed under the authority of and as part of the responsibility of the pathologist", was relayed by the French Union of Pathologists (SMP) on June 132013.

${ }^{2}$ Eliot Freidson (1970) was one of the first to argue that physicians, despite professional rhetoric, behaved not as scientist but as primarily action-oriented practitioners. 
tensions between pathologists and molecular biologists over the definition of legitimate knowledge (morphological combined with molecular biology vs. "pure" molecular biology) and expertise (physicians vs. biologists) in the characterization of cancers exhibited some features of "jurisdictional struggles" (Abbott 1988) or of "boundary work" (Gieryn 1983).

There is also the big problem of some biological engineers who are reliable, rigorous, good people, but who can't help but tell you that they could function well on their own, without pathologists. It's a caricature, but it's a bit like that. They say: "Look, the mutations, I don't need you to co-sign them because I'm sure of what I've done in my mutation." I tell them: "That's not the problem, it's just that we participated upstream, we selected the cases, we have a global vision of the case, we know if there were other molecular biomarkers that were conducted on this case, we have the synthesis in our head, we are just here to say we did this work but also to have a second pair of eyes, a verification, a global coherence." That's what they're struggling with.

Interview with a pathologist in charge of a laboratory

This interview extract shows how the pathologists were afraid of being marginalized in the genomic medicine trials. Nevertheless, on the sites $\mathrm{A}$ and $\mathrm{C}$ where biologists and clinicians attempted to completely bypass pathologists, this strategy was neither effective nor sustainable. Indeed, both genomic trials remained highly dependent on pathology laboratories to de-archive tumor specimens, to assess the area to be scraped (for intake review), and to extract DNA from the specimen so that it could be sequenced. On another site, the head of the pathology laboratory characterized his capacity to negotiate with clinician-researchers as strong, explaining that his laboratory remained what STS scholars would call an "obligatory point of passage" (Callon 1986).

You have [Translational Research] Unit X, which is very biomarker-oriented, and that's the prototype of oncologists who feel they don't need pathologists, that they're just there to piss them off and explain to them that [what they want] can't be done... So they're going over it, and they've done 10 years of pure genetics and are now thinking: 'How are we going to get back to the tumor?"

Interview with a pathologist in charge of a laboratory 
Clearly, genomics was seen to be a new option that might eventually compete with a morphological approach to cancer management. However, the production of translational research based on genomic analysis was still dependent on the intervention of pathologists, since the reliability of the mutational analysis depended on the quality of the tumor, which pathologists were alone able to assess.

For the biological part, i.e. whether there is a mutation or not, we are the ones who interpret, it's not morphological. However, morphology still plays a role in the "preanalytical" stage: we need them to tell us whether or not we are working on tumor cells. This is still important because if we don't work on tumor cells, there is no point in working on them afterwards.

Interview with a molecular biologist

As we will show in the next section, these struggles between biologists and clinicians on one side and pathologists on the other had less to do with a binary confrontation, with one expertise excluding the other from diagnostic activities, than with how the two types of knowledge were coordinated.

\section{Varieties of coordination modalities between biologists and pathologists}

Not only did we observe a diversity of coordination/cooperation schemes involving researchers - including molecular biologists - and pathologists between the different sites studied, we also observed such schemes within sites.

Firstly, on some sites, we noticed that pathologists were more involved in other translational research projects, such as proteomics or the integration of optical imaging into diagnosis, than in precision medicine. As a matter of fact, these other researchers praised the ability of pathologists to participate in research efforts, which was in stark contrast to what most medical oncologists and molecular biologists involved in precision medicine had to say. 
These collaborations contradicted their diagnosis about the innate reluctance of pathologists towards research.

So, very few pathologists have actually engaged in the personalized medicine protocols that are the pride and the international glory of [our center]. On the other hand, half of the pathologists here are engaged in experimental protocols which are much less the international glory of [our center] [i.e. genomic-driven trials], but which one day may also lead to applications. In other words, they are very much in tune with new optical imaging protocols. And here you have 3 or 4 protocols that are also very time-consuming for them.

Interview with the head of a department of pathology

I've always liked working with pathologists because they're very open and very willing, unlike some scientists, who, when presented with new technologies, are a little reluctant [...]. Anything new that can be tested, that can possibly improve things, they're willing to go for it. They're very interesting people. I've always found them to be extremely openminded people. [...] In the research and technologies we work with, we need to work with them a lot.

Interview with a Professor of Proteomics

Furthermore, by looking carefully at the local situations, we were able to observe that the exchanges between specialties in relation to precision medicine were not doomed to confrontation. They were at times reciprocal, but above all more balanced than this. On site $\mathrm{D}$, once biologists had tested and validated molecular analysis techniques on the NGS platform, they did not hesitate to train pathologists in order to be able to integrate them routinely into the program.

We've trained [pathologists], we've developed their skills... we've helped them choose what technology to use, what strategy to use for sequencing, whether it has the... the best value for money, you might say. And then we transferred the... it was a collaborative effort. [...] it was more of a collaboration in terms of implementation, and after a... we gave them the keys... we said, "Well, now we're training you on the sequencers as well" ... because in the beginning we were doing their sequencing, as it was a one-time thing, for development. And then we... They're completely autonomous on the platform now. 
On site $\mathrm{C}$, some pathologists described the good relationship they had developed around precision medicine with a clinician-researcher from site B, while being in open conflict with those of their own center. In particular, they highlighted the fact that the clinician-researcher recognized the added value of a thorough morphological analysis and, reciprocally, they found molecular analyses useful for complementing difficult cases.

In any case, what's certain is that [a pathologist colleague] and I always send cases [to this clinician-researcher at Site X] and he's always interested in our cases because we've been to the end of what we could do with morphological analyses. They fit right in with his projects, there's a real complementarity, an understanding of each other's project.

That's the ideal situation.

Interview with a pathologist

Finally, we noticed another example of collaboration between a molecular biologist and pathologists on site C. In this case, the collaboration was based on the biologist's dependence on pathologists for his analysis.

Pathology can be distinguished from molecular biology. I'm very much in between the two, in fact, because I have techniques that are quite morphological, such as the FISH [Fluorescence in situ hybridization] method, and you can never really classify that kind of technique. [...] It's funny because [the local director of the accredited site of translational research] doesn't think of me as a biopath'. [...] Instead he sees me as a CGH platform, biomol [i.e. molecular biologist]. He's always very happy with my work, but that's it. It's complicated, it's annoying because I'm really intrigued by pathologists [and he is in conflict with them]. My analysis depends entirely on their point of view. He doesn't realize that. For example, he doesn't realize that if they take out the[ir] technician [as a consequence of the conflict], there'll be a problem with my FISH analyses. Interview with a biologist

In return, this fluorescent in situ hybridization (FISH) technique, which consists of hybridizing tumor DNA to detect gene deletions, translocations or amplifications, is a 
molecular biology technique that pathologists use for morphological analysis.

For me, FISH, which is molecular in the sense that we're interested in DNA, is still a morphological technique, although it's done by people like $[\mathrm{X}]$ who are molecular biologists. We can see that it's all interconnected and that there is a logic to the fact that the chain is homogeneous.

Interview with the technician

With regard to genomics trials, we observed pathologists readily agreeing to participate in some of them, even when their expertise in tumor morphology was not involved in inclusion decisions and even when their involvement was limited to the low-value task of measuring the tumor percentage of samples. This involvement was mainly based on three conditions: 1) they anticipated that they would regain control over the "arc of diagnosis" (to refer to the notion of the "arc of work" proposed by Strauss and colleagues [1985]) if certain elements evaluated in the trial became routine (the platform biologists would thus only intervene on a punctual basis); 2) the trial should respect certain organizational conditions that made it possible without too much difficulty to anticipate a change in routine activity; 3) the timescales and the volume of specimens to be processed during the trial should not be too disruptive to the normal operation of the laboratory.

You can see that you can't create that in a small research shop [sic] because, after that, the industrial workflow won't be adapted. [...] If we develop in a research area that lives in another mode, not world, mode, which is more in project, experimental mode, with experimental tools that don't have interfaced IT tools, that don't manage patient identity in the same way, that don't manage the same constraints of permanent diagnosis and care, we won't be able to implement these tools in our department. Interview with a technician in charge of the management of a Department of Pathology

Finally, interprofessional relationships should not be interpreted as mere cognitive/epistemic struggles between disciplines fighting for the control of peculiar jurisdictions, but should rather be analyzed as following from interdependences that link actors of different disciplines 
for the joint implementation of work activities. Interprofessional relationships also depended on the degree of disruption to the routine organization of work that these translational research projects could cause in pathology laboratories (i.e. they were based on practices but also on stabilized relationships between actors). Indeed, we will now detail how translational research related to precision medicine was difficult to integrate in laboratories whose production had been rationalized.

\section{Doing translational research in a rationalized department}

Pathology has become a key activity in modern hospitals (Keating and Cambrosio 2003). In order to cope with a growing demand for procedures since the 1960s (Rothstein 1979), in terms of initial diagnosis, prognosis, and continuous evaluation of the effectiveness of treatments, pathology departments have continuously tried to rationalize their activity. This move towards rationalization has translated into a constantly renewed division of labor, and has been reinforced by the development of new technologies and the increased use of machines for repetitive and standard tasks capable of enhancing the reliability of the results rendered (Vézian 2019). Today's pathology departments are thus complex organizations within which, alongside medical and technical functions, management functions aim at supervising this sophisticated division of labor, at ensuring technical supervision of machines, and at controlling the resources needed to carry out tasks. This high degree of organizational and practical sophistication has complicated the involvement of pathologists in precision medicine.

\section{The requirements of an industrialized activity of diagnosis and prognosis}

Because of their routine diagnostic activity, pathologists are (still) custodians of tumor specimens, which are basic to all genomic analysis. As such, they play a central role in the management of the sampling and provision circuit, which may be used in translational 
research or even fundamental research.

Pathologists must comply with a set of forensic constraints. For example, they must archive specimens and histopathological microscopic documents that enable them to establish a diagnosis, whether or not this reveals a cancer. This aspect has led them to be particularly vigilant about the proper use of tumor specimens, and they do not hesitate to issue a veto if they find that the specimen is too small to be used for research purposes. The provision of specimens (blocks, slides, or frozen fragments) is subject to various legal and ethical regulations. These include an informed consent form signed by the patient in which he or she accepts that samples collected for health purposes may also be used for research purposes (under certain conditions). However, the development of targeted therapies based on massive data analysis has necessitated developing new modes of consent (which we cannot go into detail here at the risk of complicating our subject). As a consequence of all these new requirements, implementation of precision medicine projects can be slowed down. The due acknowledgment of these administrative and medico-legal constraints by biologists and clinician-researchers influenced the good will of pathologists in committing themselves (or not) to precision medicine projects on the sites we studied. For example, on site B, the precision medicine actors had thought carefully about a new consent form, which allowed for the use of the same biological material for several research projects. Since this initiative met pathologists' norms and requests, cooperation was made easier.

Another factor related more specifically to the performance of analyses required for genomic testing. It was easier for pathologists to process it when the sample was new or, at least, when it had not been processed in the past. It might be then inserted into the traditional diagnostic workflow more smoothly. Conversely, when the trial used previously analyzed tumor material, the more the number of samples to be processed increased, the more the 
workflow would be disrupted. This is what happened in the center that decided to stop patient accrual for three months (see above).

When you look for [the tumor blocks], you have to look for them somewhere else, it's not in the regular storage. It means that we start putting our eggs everywhere, and very quickly, given the volumes we produce, that can be a source of difficulty. If everyone started doing that, it wouldn't be manageable. Or we'd need as many labs as we have projects!

Interview with a technician in charge of the management of a Department of Pathology

Having a DNA collection in a freezer available, versus taking a sample from the pathologist's macroscopy room via extraction to the accredited sequencer. The sampling circuit is really a very cumbersome thing when there is volume and response time. Interview with a Pathologist

Another aspect related to the division of labor that was required to carry out these analyses. Regardless of the qualities of the tumor specimen, this increased the volume of activity and could generate additional needs in terms of personnel (physicians and technicians) that appeared difficult to fulfill. In addition to the small number of pathologists trained in molecular techniques in France, the lack of medical and technical resources in the pathology departments influenced the way pathologists considered the new trials. Although the routine organization of the pathology department was characterized by systematic standardization through the regular purchase of machines, certain tasks could not be the subject of such an automatic process. This was the case for cutting the block (i.e. the tissue embedded in paraffin).

This was of course case-specific, as the specimen was necessarily different from one patient to another, and also increasingly smaller as the specimen was processed. It was therefore required that the technician assigned to the precision medicine research project be experienced. Paradoxically, the expertise accumulated through routine, morphological diagnostic activity proved to be decisive with regard to the preparation of tumor material 
before NGS processing for research purposes. This situation not only disrupted the department's production, but also led to conflicts over the staff assigned to the trial.

It's true that we could say, "Let's take a technician $\mathrm{X}$ or $\mathrm{Y}$, a technician that we hire for this research project and then it won't be your technicians who will do the job, it will be the new technician." But these new technicians need to be trained. And then, an increasingly complicated barrier is that the samples are getting smaller and smaller, so it takes dexterity to handle them. You can't ask a technician who has never cut a microtome, never scraped a sample, who doesn't know what it's like to select a tumor zone that's less than a millimeter in size.

Interview with a pathologist

The pressure on departmental resources that participation in translational research implied was all the less willingly accepted by these laboratories given that the general funding system of French hospitals, based for some years now on North-American DRGs, provide an incentive to produce care. It made it more difficult for specialties such as pathology, from whom the management required high productivity, to participate in research. It is true that the "reference system for innovative acts outside nomenclature" (RIHN), a new support system for innovation in biology and pathology, has been in place since 2015. However, in this nomenclature, which lists the acts for which there exists a rating associated with a given set of funding, not all molecular techniques are included. Thus, significant costs have remained for healthcare institutions, and for individual departments.

We're drowning in these things [i.e. research projects regarding precision medicine], knowing that we've had this [molecular] approach for years, without funding! Everything I've told you about expertise, with all the biomol [i.e. molecular biology]... Zero! Zero funding by social security! That's why they're starting to pout... We have regular meetings with the CFO and the CEO to find out how we can move forward on this. It's not every week but we had two meetings in the last month. It's a real, real problem. We spend a lot and we don't make much money.

Interview with a Pathologist 
Thus, the economic context of the health care system indirectly influenced the relationship between pathologists and those involved in precision medicine.

\section{The need to maintain stable cooperative networks with external pathologists}

In the academic institutions where translational research projects related to precision medicine were carried out, the activity of the pathologists was not only about producing diagnoses in quantity, they were also depended on for their expertise. This expertise was conditioned by their ability to obtain from their colleagues both in private practice and in community hospitals a regular supply of tumor specimens (for a second opinion, for instance) in order to enhance their expertise regarding specific, complex pathologies.

However, the implementation of precision medicine contributed to destabilizing already delicate interactions. Some genomic-driven trials required returning to the specimens being treated in the initial treatment centers and therefore requesting tumor material from forprofit pathology laboratories or public departments located in community hospitals. The provision of a specimen represented a cost for pathology departments, and for-profit laboratories were particularly sensitive to this. Although there was a small compensation for the provision of tumor specimens, this was considered to be too low. At the time of our research, the tensions engendered by these interactions were all the more tense given that the public authorities refused to set up molecular genetics platforms in the private sector, forcing private pathologists to hand over their specimens and the information collected to the public hospitals.

We are assailed every day with requests for blocks, requests for material, requests to send blocks, whether for INCa studies or for industrial studies. This is very frowned upon today by our colleagues in the private sector, who have the impression that they are wasting their time, and it frankly disturbs their activity. Because we have the impression from a distance that it is an activity of the "I'm going to look for something in an archive" type. It's not like that at all! First of all, I'm going to look for a needle in a haystack 
because we're going to look for a block among the 50 blocks of the patient for today's operation, we're going to look for the tumor block, so we'll have to re-select it. We're talking about very high numbers.

Interview with a manager of a pathology department

Precision medicine therefore posed a double threat to the network of collaborative relationships that pathologists had patiently and carefully developed in order to carry out their work, including both relationships within and outside the department.

\section{Organizational innovations}

Initiatives had been taken locally in order to reorganize work and respond to some of these difficulties. In the center where the conflict had led to a three-month break in patient accrual, the genomics trial managers, in consultation with the pathologists, learned from this difficult experience and adopted a rule to regulate the workflow: no medical oncologist in the cancer center could accrue more than ten patients per week. This type of organizational rule was very unusual in a trial. They also experimented with a new work process, entrusting the biobank with the extraction of DNA. But this was not enough to free the researchers from dependence on pathologists, since the determination of the relevant area to be extracted remained under the pathologists' expert control.

You always come up against the pathologist, the pathologist's grip... being forced in that particular trial to have an area to be determined to do the scraping and extraction [...] That's the sticking point and that could slow down the protocol as well. Head of the biobank.

Finally, the PI asked the clinical trial management unit to dispatch a technician to the pathology department in order to take charge of all logistics related to the management of tumor specimens, which relieved the department managers of this task.

Another PI in site A created and funded a new platform for experimental pathology, 
formally situated inside the pathology department but relieved from all activities related to routine care. While it helped to smooth relationships between the head of the department and the medical oncologists involved in precision medicine research projects, the head of the department still regretted that the physician in the experimental platform did not take part in the diagnostic activities when there were peaks of activity, in a context of increasing scarcity of medical time.

Cancer centers have always had problems with their pathology departments. Because they're not molecular pathologists, they missed a step. So we created a department of molecular pathology out of thin air [inside the department]. I was tired of their infighting, I didn't want to have anything to do with that anymore.

Medical oncologist, Head of an accredited site for translational research

On site B, the head of the department experimented with the implementation of lean management techniques in order to increase productivity on routine tasks and free up resources for less standardized work. But s-he had to back pedal very fast, when s-he realized that purely industrial methods were not suitable for the hospital's operation.

We can see from these examples that, while the problems involved in integrating pathologists in precision medicine and coordinating their diagnostic activity with translational research projects were not solved, organizational solutions continued to be experimented with.

\section{Conclusion}

Precision medicine involves an intertwining of care and research, beyond even what the relevant actors anticipated. In France, pathologists have not been thought of as relevant actors in translational research and, as a result, have not been involved prior to the launch of projects such as genomic medicine trials. However, we have seen the extent of the implications that these projects have for the activity of pathologists, whatever their prior experience with 
"traditional" clinical and basic research. Consequently, new ways of collaborating between disciplines and of organizing work in the laboratories have had to be tried out. The extent to which epistemic innovations such as precision medicine require organizational experimentation is clear.

Several substantive conclusions can be drawn from these examples and empirical data. They have implications for organizational sociology, sociology of professions, and STS. It is clear that cooperation and conflict can be better explained by taking the structuring role of the constraints of collective action seriously. What impedes (and at other times fosters) cooperation between professionals has less to do with: a) jurisdictional competition in which a professional or expert group strives to exclude other groups from their professional remit; and/or b) differences (or consistency) in axiological, cognitive, and epistemic beliefs and representations. Both cooperation and conflict are better understood as a consequence of: a) the matching and coordinating (or mismatching and leaving uncoordinated) of professional practices, in networks where some actors control the "arc of work" (Strauss, 1985) and others agree to supply them with specific services, and/or b) the necessity of establishing and maintaining stable networks of providers and recipients.

\section{References}

Abbott, A. 1988. The System of Professions: An Essay on the Division of Expert Labor. Chicago: Chicago University Press.

Amsterdamska, O. 2005. "Demarcating Epidemiology." Science, Technology, \& Human Values 30 (1): 17-51.

Anteby, M., C. K. Chan, and J. DiBenigno. 2016. "Three Lenses on Occupations and Professions in Organizations: Becoming, Doing, and Relating." The Academy of Management Annals 10: 183-244.

Beaudevain, C., A. Peerbaye, and C. Bourgain. 2018. "'It has to become true genetics': tumour genetics and the division of diagnostic labour in the clinic." Sociology of Health \& Illness 41 (4): 643-657.

Bourret, P., and A. Cambrosio. 2019. "Genomic expertise in action: molecular tumour boards and decision-making in precision oncology." Sociology of Health \& Illness 41: 1568-1584. Bucher R. 1962. "Pathology: A Study of Social Movements within a Profession", Social Problems 10(1): 40-51. 
Callon, M. 1986. “Éléments pour une sociologie de la traduction.” L'Année sociologique (36): 169-208.

Cambrosio, A., P. Keating, E. Vignola-Gagné, S. Besle, and P. Bourret. 2018. "Extending experimentation: oncology's fading boundary between research and care." New Genetics and Society 37 (3): 207-226.

Close-Koenig, T. 2007. "A detour or a shortcut? Pathology laboratories in cancer treatment centres." In Transferring Public Health, Medical Knowledge, and Science in the 19th and 20th Century, edited by A. Andresen and T. Grønlie, 47-65. Rokkansenteret: Report 2.

Crabu, S. 2018. "Rethinking biomedicine in the age of translational research: Organisational, professional, and epistemic encounters." Sociology Compass 12: e12623.

Freidson, E. 1970. Profession of Medicine. A Study of the Sociology of Applied Knowledege. New York: Dodd and Mead.

Gieryn, T. F. 1983. "Boundary Work and the Demarcation of Science from Non-Science:

Strains and Interests in Professional Ideologies of Scientists." American Sociological Review 48 (6): 781-795.

Green, S. 2019. "Plastic diagnostics: The remaking of disease and evidence in personalized medicine." Social Science \& Medicine (published online before print).

Hendriks B., A. Simons, and M. Reinhart. 2019. "What are Clinician Scientists Expected to do? The Undefined Space for Professionnalizable Work in Translational Biomedicine", Minerva 57: 219-237.

Keating, P., and A. Cambrosio 2003. Biomedical Platforms. Realigning the Normal and the Pathological in Late-Twentieth-Century Medicine. London: The MIT Press.

Kerr, A., J. Swallow, C. K. Chekar, and S. Cuningham-Burley. 2019. "Genomic research and the cancer clinic: uncertainty and expectations in professional accounts." New Genetics and Society 38 (2): 222-239.

Lamont, M., and V. Molnár 2002. "The Study of Boundaries Across the Social Sciences." Annual Review of Sociology 28: 167-195.Löwy, I. 1996. Between Bench and Bedside. Science, healing and interleukine-2 in a cancer ward, Cambridge, London, Harvard University Press.

Nelson, N., P. Keating, A. Cambrosio, A. Aguilar-Maheca, and M. Basik. 2014. "Testing devices or experimental systems? Cancer clinical trials take the genomic turn." Social Science \& Medicine 111: 74-83.

Rothstein W.G. 1979. "Pathology: the evolution of a specialty in American medine." Medical Care 17 (10): 975-988.

Strauss, A. 1985. Work and the Division of Labor. The Sociological Quarterly 26 (1): 1-19. Vézian A. 2019. "Le rôle contesté des pathologists dans la médecine de précision." In Les politiques de lutte contre le cancer en France, edited by P. Castel, J.-A. Juven and A. Vézian, 99-117. Rennes: Presses de l'EHESP.

Wilson-Kovacs D. M., and C. Hauskeller 2012. "The Clinician-Scientist: Professional Dynamics in Clinical Stem Cell Research", Sociology of Health \& Illness 34(4): 497-512.

Wright, S., M. Porteous, D. Stirling, O. Young, C. Gourley, and N. Hallowell. 2019.

"Negotiating jurisdictional boundaries in response to new genetic possibilities in breast cancer care: The creation of an 'oncogenetic taskscape"”, Social Science \& Medicine 225: 26-33.

\section{Count: 6539 words.}

